This document is the Accepted Manuscript version of a Published Work that appeared in final form in Journal of the American Chemical Society, copyright (C) American Chemical Society after peer review and technical editing by the publisher. To access the final edited and published work see http://pubs.acs.org/doi/abs/10.1021/ja5064586

\title{
Ni-catalyzed Carboxylation of Unactivated Primary Alkyl Bromides and Sulfonates with $\mathrm{CO}_{2}$
}

\author{
Yu Liu ${ }^{\dagger}$, Josep Cornella ${ }^{\dagger}$ and Ruben Martin* ${ }^{\dagger \S}$ \\ ${ }^{\dagger}$ Institute of Chemical Research of Catalonia (ICIQ), Av. Països Catalans 16, 43007, Tarragona, Spain \\ ${ }^{\S}$ Catalan Institution for Research and Advanced Studies (ICREA), Passeig Lluïs Companys, 23, 08010, Barcelona, Spain
}

Supporting Information Placeholder

ABSTRACT: A Ni-catalyzed carboxylation of unactivated primary alkyl bromides and sulfonates with $\mathrm{CO}_{2}$ at atmospheric pressure is described. The method is characterized by its mild conditions and a remarkable wide scope without the need for air- or moisture-sensitive reagents, thus becoming a user-friendly and operationally simple protocol en route to carboxylic acids.

The utilization of $\mathrm{CO}_{2}$ as an alternative renewable feedstock has recently received a significant attention in the scientific community. ${ }^{1}$ Such interest is primarily associated to the fact that $\mathrm{CO}_{2}$ is nontoxic, abundant and nonflammable, hence constituting an opportunity for carbon sequestration and allowing the implementation of innovative, yet practical, methodologies counterintuitive at first sight. ${ }^{1}$ Beyond any doubt, the synthesis of carboxylic acids represents an ideal target in $\mathrm{CO}_{2}$ fixation since a myriad of molecules such as Atorvastatin, Beraprost, Artesunate, Pemetrezed or Pregabalin, among others, display a significant biological activity (Scheme 1). ${ }^{2,3}$

Scheme 1. Biological Significance of Carboxylic Acids.

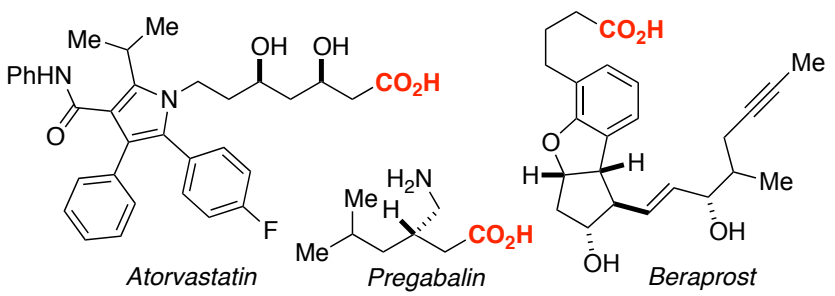

Encouraged by a seminal work of Osakada and Yamamoto, ${ }^{4} \mathrm{we}^{5}$ and others ${ }^{6}$ launched a program aimed at unlocking the potential of $\mathrm{CO}_{2}$ in reductive catalytic reactions (Scheme 2, path a). ${ }^{7}$ Unlike carboxylation events based on stoichiometric, well-defined and, in some cases, air-sensitive organometallic species (path b), ${ }^{8,9}$ such reductive events offer higher flexibility and ease of execution by using simpler building blocks, thus representing an added value from a simplicity, reliability and step-economical standpoint. Unfortunately, reductive carboxylation protocols are inherently restricted to substrates that rapidly undergo oxidative addition such as aryl ${ }^{5,6}$ or benzyl halides (path a). ${ }^{5 a, 5 b}$ Ideally, this field should include the use of unactivated alkyl electrophiles possessing $\beta$-hydrogens. Indeed, these substrates are the most challenging in the cross-coupling arena due to their reluctance towards oxidative addition and the proclivity of in situ generated alkyl metal species for $\beta$-hydride elimination, homodimerization or hydrogen abstraction pathways, among others. ${ }^{10}$ Therefore, at the outset of our investigations it was unclear whether a metalcatalyzed carboxylation event could ever be conducted with unactivated alkyl electrophiles. ${ }^{11}$ If successful, such a process would offer an unrecognized opportunity in $\mathrm{CO}_{2}$ fixation while opening up new possibilities via unconventional bond disconnections. Herein we report a mild Ni-catalyzed carboxylation of unactivated primary alkyl bromides and sulfonates possessing $\beta$-hydrogens with $\mathrm{CO}_{2}$ (path c). The protocol represents a convenient method to rapidly access carboxylic acids from simple precursors without handling air-, moisture-sensitive reagents or cyanide sources and it is characterized by a wide scope and an excellent chemoselectivity profile.

Scheme 2. Reductive Carboxylation Reactions with $\mathrm{CO}_{2}$. 


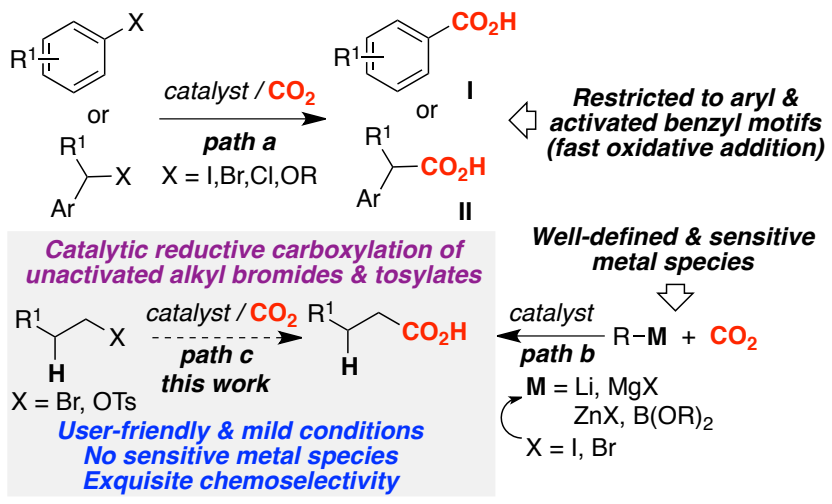

We initiated our investigations with 1a as the model substrate with $\mathrm{CO}_{2}(1 \mathrm{~atm})$ at room temperature (Table 1 ). As expected, the conditions previously employed for the carboxylation of aryl halides ${ }^{5 \mathrm{c}, 6}$ or primary benzylic halides $^{5 a, 5 b}$ failed to convert 1a into $2 a$. Initial screening of metal complexes identified $\mathrm{NiCl}_{2} \cdot$ glyme as a competent catalyst with cheap $\mathrm{Mn}$ as reducing agent. ${ }^{12}$ While nitrogen donors have successfully been employed as ligands in cross-coupling reactions of unactivated alkyl halides, ${ }^{13}$ no conversion to $\mathbf{2 a}$ was observed with commonly employed bipyridines, tert-pyridines or oxazolines (L1-L9). ${ }^{12}$ In these cases, dimerization, $\beta$ hydride elimination and recovered starting material was observed in the crude reaction mixtures. A similar reactivity pattern was found when employing simple phenanthroline-type ligands (L10-L13). We speculated that an increase in the steric bulk around the nitrogen-donor ligand could lead to more robust Ni complexes with enhanced stability and greater activity. In line with this notion, we found that $\mathbf{L 1 4}$ delivered $\mathbf{2 a}$ in $66 \%$ yield. Analogously, L16, a bench-stable ligand readily obtained in one-step and in bulk quantities, ${ }^{14}$ allowed for obtaining 2a in a $76 \%$ isolated yield. Dimerization and traces of $\beta$-hydride elimination byproducts account for the observed mass balance. ${ }^{15}$ Importantly, the reaction could be scaled up without any erosion in yield. Intriguingly, subtle changes in the electronic or steric environment of the 1,10-phenanthroline backbones had a deleterious effect (L15 and L17). ${ }^{16}$ Control experiments unambiguously revealed that all reaction components were necessary to promote the carboxylation of $\mathbf{1 a} .^{12,17}$

Table 1. Ligand influence on the reaction outcome. ${ }^{a, b}$

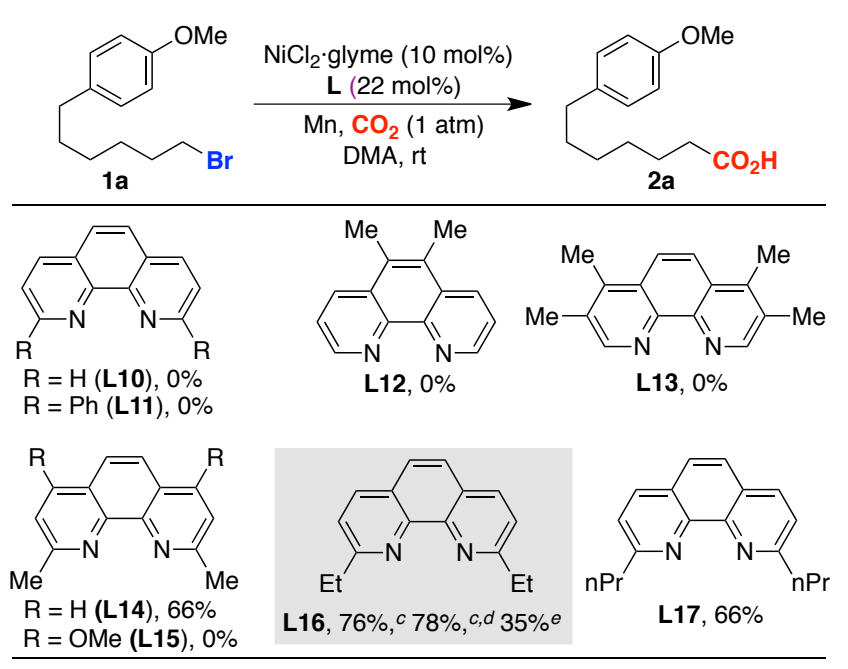

${ }^{a}$ Reaction conditions: 1a $(0.15 \mathrm{mmol}), \mathrm{NiCl}_{2} \cdot$ glyme $(10$ mol\%), L (22 mol\%), Mn (0.33 mmol), DMA (0.15 M), at rt under $\mathrm{CO}_{2}(1 \mathrm{~atm})$ for $12 \mathrm{~h} .{ }^{b}$ Yields were determined by HPLC analysis using naphthalene as internal standard. Isolated yield. ${ }^{d} \mathbf{1 a}(1.0 \mathrm{mmol}) .{ }^{e} \mathrm{NiCl}_{2} \cdot$ glyme $(5 \mathrm{~mol} \%)$.

Table 2. Ni-catalyzed Carboxylation of Alkyl Bromides. ${ }^{a, b}$ 


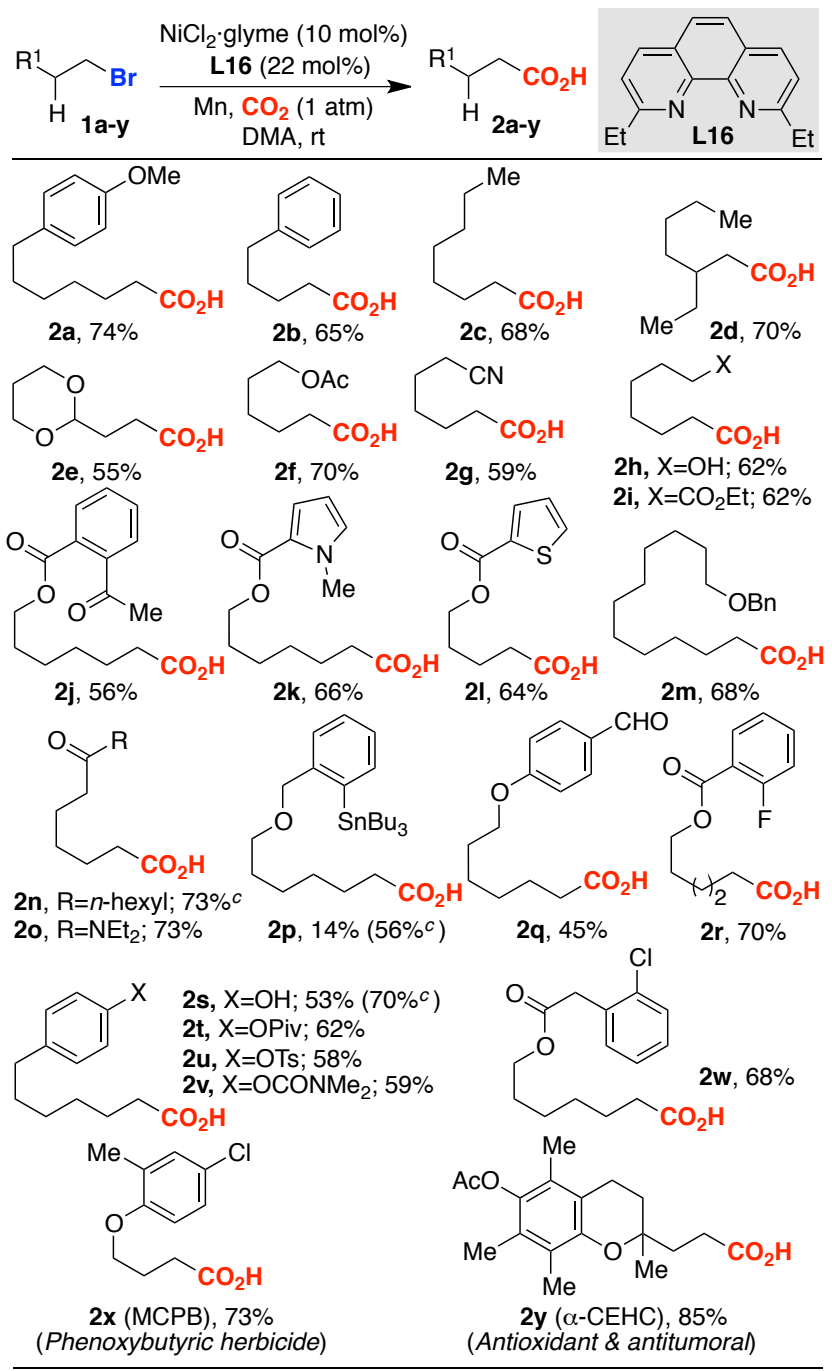

${ }^{a}$ Reaction conditions: 1a-u $(0.30 \mathrm{mmol}), \mathrm{NiCl}_{2} \cdot$ glyme (10 mol\%), L16 (22 mol\%), Mn (0.66 mmol), DMA (0.15 M), at $\mathrm{rt}$ under $\mathrm{CO}_{2}(1 \mathrm{~atm})$ for $12 \mathrm{~h} .{ }^{b}$ Isolated yields, average of at least two independent runs. ${ }^{c}$ Using L14 (22 mol\%).

Encouraged by these findings, we set out to explore the preparative scope of our reaction. As shown in Table 2, a host of unactivated primary alkyl bromides possessing $\beta$-hydrogens could be equally accommodated in good yields. ${ }^{18,19}$ Particularly illustrative is the chemoselectivity profile of our protocol as esters (2f, 2i-l, 2r, 2t, 2w and $\mathbf{2 y}$ ), nitriles $(\mathbf{2 g})$, heterocycles ( $\mathbf{2 k}$ and $\mathbf{2 l}$ ), acetals (2e), amides (2o), ketones (2j and $\mathbf{2 n})$ and even aldehydes (2q) were tolerated. Notably, unprotected aliphatic alcohols (2h), phenols (2s) or carbonyl compounds containing relatively acidic $\alpha$-protons $(\mathbf{2 g}, \mathbf{2 i}, \mathbf{2 j}, \mathbf{2 n}, \mathbf{2 o}$ and $\mathbf{2 w}$ ) did not compete with the efficacy of the carboxylation event. At the current level of development, unactivated secondary alkyl bromides cannot be employed as coupling partners. ${ }^{19}$ Surprisingly, the reaction could also be conducted in the presence of aryltin reagents (2p) with L14, thus providing ample opportunities for subsequent manipulation. Interestingly, no macrocycle resulting from an intramolecular addition of the ar- yltin into the $\mathrm{C}\left(\mathrm{sp}^{3}\right)-\mathrm{Br}$ bond or a carboxylation event on the $\mathrm{C}-\mathrm{Sn}$ bond were detected in the crude material. ${ }^{19}$ While conformational restrictions might account for the former, the latter is particularly interesting since organotin reagents have been reported to efficiently undergo carboxylation events. ${ }^{20}$ Similarly, $\mathbf{L 1 4}$ provided better results for $\mathbf{2 s}$. Site-selectivity could be accomplished in the presence of electrophilic sites amenable for Nicatalyzed cross-coupling reactions such as aryl pivalates $(\mathbf{2 t}),{ }^{21}$ acetates $(\mathbf{2 y}),{ }^{21}$ carbamates $(\mathbf{2 v})^{21}$ or aryl fluorides (2r). ${ }^{22}$ While aryl chlorides, ${ }^{6 a}$ tosylates $^{6 a}$ or pivalates ${ }^{5 a}$ have been used in reductive carboxylation reactions, we found exclusive $\mathrm{CO}_{2}$ insertion into the $\mathrm{C}\left(\mathrm{sp}^{3}\right)-\mathrm{Br}$ bond $(2 t, 2 u-2 x)$. The synthetic value of this transformation is illustrated by a concise synthesis of compounds that exhibit potent biological activities such as MCPB (2x) and $\alpha$-CEHC (2y) from available precursors. ${ }^{12}$

Table 3. Ni-catalyzed Carboxylation of Alkyl Sulfonates. ${ }^{a, b}$

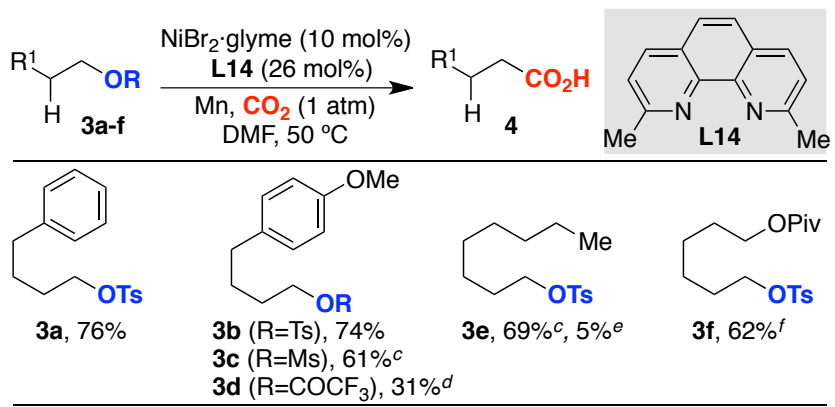

a 3a-f (0.30 mmol), $\mathrm{NiBr}_{2} \cdot \mathrm{glyme}(10 \mathrm{~mol} \%), \mathbf{L 1 4}$ (26 mol\%), Mn (2.4 equiv), DMF $(0.15 \mathrm{M})$ at $50{ }^{\circ} \mathrm{C}$ for $12 \mathrm{~h}$. ${ }^{b}$ Isolated yields, average of at least two independent runs. ${ }^{c} 60{ }^{\circ} \mathrm{C} .{ }^{d} 100{ }^{\circ} \mathrm{C} .{ }^{e} 7.5 \mathrm{~mol} \% \mathrm{NiBr}_{2} \cdot$ glyme. ${ }^{f} 70{ }^{\circ} \mathrm{C}$.

In light of these results we wondered whether we could extend our Ni-catalyzed reductive carboxylation event to unactivated alkyl sulfonates. While the reaction of $\mathbf{3 b}$ under the optimized conditions for alkyl bromides (Table 2) resulted in lower conversions to products, the combination of $\mathrm{NiBr}_{2} \cdot$ glyme, $\mathbf{L 1 4}$ and DMF as the solvent at $50{ }^{\circ} \mathrm{C}$ under $1 \mathrm{~atm} \mathrm{CO}_{2}$ was optimal, furnishing the corresponding carboxylic acid in $76 \%$ yield. ${ }^{12,14}$ Interestingly, alkyl mesylates (3c) or trifluoroacetates (3d) could also be employed, albeit in lower yields. Notably, the presence of other C-O electrophiles such as alkyl pivalates did not interfere, resulting in the selective carboxylation of the alkyl sulfonate backbone (3f). Overall, we believe the results in Tables 2 and 3 shows the robustness and the prospective impact of our Ni-catalyzed carboxylative protocol when employing unactivated alkyl bromides or alkyl sulfonates. ${ }^{23}$

Although an in depth mechanistic study should await further investigations, we wondered whether the reaction was initiated by $\beta$-hydride elimination followed by a hydrocarboxylation event. ${ }^{24}$ To such end, we subjected 5-phenyl pentene (5) under our optimized conditions. 
Under the limits of detection, we did not detect any carboxylation reaction. ${ }^{12} \mathrm{~A}$ similar result was obtained when exposing $n$-butylMnBr (6) to our $\mathrm{Ni} / \mathbf{L 1 6}$ system in the presence or absence of $\mathrm{Mn}$, thus leaving some doubt about the intermediacy of organomanganese species. ${ }^{12}$ In order to shed light into the mechanism, we decided to study the carboxylation reaction of $7 \mathbf{a}$ and $7 \mathbf{b}$ (Scheme 3). ${ }^{12}$ A diastereomerically pure 8 was anticipated for a mechanism consisting of a "classical" oxidative addition; ${ }^{25}$ on the contrary, a statistical mixture of diastereoisomers in $\mathbf{8}$ would account for a free-radical mechanism via single electron transfer (SET). As shown in Scheme $3,{ }^{1} \mathrm{H}-\mathrm{NMR}$ spectroscopical analysis of the crude mixture revealed the loss of stereochemical integrity at $\mathrm{C} 1{ }^{26}$ A similar behavior was found for $\mathbf{7 c}$ and $\mathbf{7 d}$, an observation that might indicate a scenario consisting of SET processes via $\mathrm{Ni}(\mathrm{I})$ species. ${ }^{27-31}$ In line with this notion, we observed that radical clocks such as (bromomethyl)cyclopropane and 1-bromo-5-hexene resulted in ring-opened dimerization products.

Scheme 3. Mechanistic experiments.

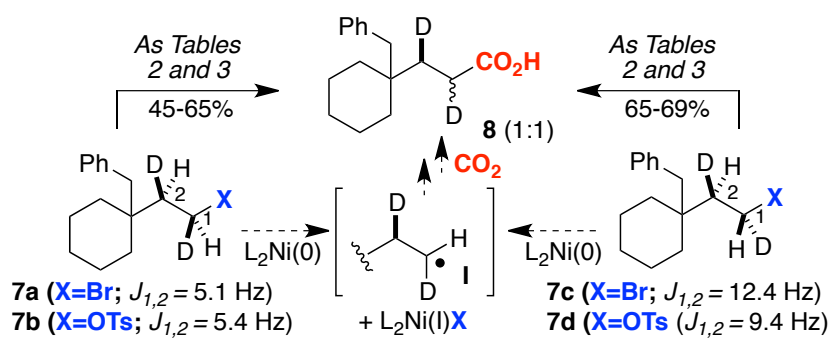

In summary, we have reported a new catalytic carboxylation of unactivated primary alkyl bromides and sulfonates possessing $\beta$-hydrogens with $\mathrm{CO}_{2}$ that gives access to valuable carboxylic acids. This method is characterized by its exquisite functional group compatibility, mild conditions, readily availability of the starting materials and ease of execution without the need for airor moisture-sensitive materials. Further investigations into the mechanism and the extension to more challenging substrate combinations are currently underway.

\section{ASSOCIATED CONTENT}

Supporting Information. Experimental procedures and spectral data. This material is available free of charge via the Internet at http://pubs.acs.org.

\section{AUTHOR INFORMATION}

\section{Corresponding Author}

*rmartinromo@iciq.es

\section{Funding Sources}

No competing financial interests have been declared.

\section{ACKNOWLEDGMENT}

We thank ICIQ, the European Research Council (ERC277883) and MINECO (CTQ2012-34054) for support.
Johnson Matthey, Umicore and Nippon Chemical Industrial are acknowledged for a gift of metal \& ligand sources. Y.L. and J.C thank COFUND and European Union (FP7PEOPLE-2012-IEF-328381) for a fellowship. This paper is dedicated to the memory of Prof. Gregory L. Hillhouse.

\section{REFERENCES}

(1) For selected reviews, see: (a) Zhang, L.; Hou, Z. Chem. Sci. 2013, 4, 3395. (b) Tsuji, Y.; Fujihara, T. Chem. Commun. 2012, 48, 9956. (c) Cokoja, M.; Bruckmeier, C.; Rieger, B.; Herrmann, W.A.; Kühn, F. E. Angew. Chem., Int. Ed. 2011, 50, 8510. (d) Martin, R.; Kleij, A. W. ChemSusChem 2011, 4, 1259. (e) Huang, K.; Sun, C.-L.; Shi, Z.-J. Chem. Soc. Rev. 2011, 40, 2435. (g) Sakakura, T.; Choi, J.-C.; Yasuda, H. Chem. Rev. 2007, 107, 2365

(2) (a) Patai, S. The chemistry of acid derivatives. Germany: Wiley, 1992. (b) Goossen, L. J.; Rodríguez, N.; Goossen, K. Angew. Chem., Int. Ed. 2008, 47, 3100.

(3) Maag, H. Prodrugs of carboxylic acids. New York: Springer, 2007.

(4) Osakada, K.; Sato, R.; Yamamoto, T. Organometallics 1994, 13, 4645

(5) (a) Correa, A.; León, T.; Martin, R. J. Am. Chem. Soc. 2014, 136, 1062. (b) León, T.; Correa, A.; Martin, R. J. Am. Chem. Soc. 2013, 135, 1221. (c) Correa, A.; Martin, R. J. Am. Chem. Soc. 2009, 131, 15974

(6) (a) Fujihara, T.; Nogi, K.; Xu, T.; Terao, J.; Tsuji, Y. J. Am. Chem. Soc. 2012, 134, 9106. (b) Tran-Vu, H.; Daugulis, O. ACS Catal. 2013, 3, 2417.

(7) For electrochemical carboxylative processes of organic halides, see for example: (a) Ohkoshi, M.; Michinishi, J.Y.; Hara, S.; Senboku, H. Tetrahedron 2010, 66, 7732. (b) Amatore, C.; Jutand, A.; Khalil, F.; Nielsen, M. F. J. Am. Chem. Soc. 1992, 114, 7076

(8) For a review, see: Correa, A.; Martin, R. Angew. Chem., Int. Ed. 2009, 48, 6201

(9) For selected carboxylation of alkyl organoboranes: (a) Ohishi, T.; Zhang, L.; Nishiura, M.; Hou, Z. Angew. Chem., Int. Ed. 2011, 50, 8114. (b) Ohmiya, H.; Tanabe, M.; Sawamura, M. Org. Lett. 2011, 13, 1086. For the carboxylation of alkyl organozincs: (c) Ochiai, H.; Jang, M.; Hirano, K.; Yorimitsu, H.; Oshima, K. Org. Lett. 2008, 10, 2681.

(10) For selected reviews using unactivated alkyl halides: (a) Kambe, N.; Iwasaki, T.; Terao, J. Chem. Soc. Rev. 2011, 40, 4937. (b) Hu, X. Chem. Sci. 2011, 2, 1867. (c) Jana, R.; Pathak, T. P.; Sigman, M. S. Chem. Rev. 2011, 111, 1417.

(11) For photoinduced events with excess of $\mathrm{Sm} / \mathrm{SmI}_{2}$ using tungsten lamps under $h v$, see: Nomoto, A.; Kojo, Y.; Shiino, G.; Tomisaka, Y.; Mitani, I.; Tatsumi, M.; Ogawa, A. Tetrahedron Lett. 2010, 51, 6580.

(12) See Supporting Information for details

(13) Selected references: (a) Everson, D. A.; Jones, B. A.; Weix, D. J. J. Am. Chem. Soc. 2012, 134, 6146. (b) Choi, J.; Fu, G. C. J. Am. Chem. Soc. 2012, 134, 9102. (c) Binder, J. T.; Cordier, C. J.; Fu, G. C. J. Am. Chem. Soc. 2012, 134, 17003. (d) Phapale, V. B.; Guisán-Ceinos, M.; Buñuel, E.; Cárdenas, D. J. Chem. Eur. J. 2009, 15, 12681. (e) Phapale, V. B.; Buñuel, E.; García-Iglesias, M.; Cárdenas, D. J. Angew. Chem. Int. Ed. 2007, 46, 8790. (f) Powell, D. A.; Maki, T.; Fu, G. C. J. Am. Chem. Soc. 2005, 127, 510. (g) Powell, D. A.; Fu, G. C. J. Am. Chem. Soc. 2004, 126, 7788.

(14) Pijper, P. J.; Van der Goot, H.; Timmerman, H.; Nauta, W. T. Eur. J. Med. Chem. 1984, 19, 399.

(15) Lowering down the loading of the reaction components for the carboxylation of 1a was not successful. A lower Ni:L ratio resulted in higher amounts of dimerization. At present 
we believe that a $\mathrm{L}: \mathrm{Ni} \geq 2$ is needed to stabilize the resting state of the active $\mathrm{Ni}(0)$ catalyst while avoiding undesired pathways. A similar Ni:L effect has been observed in other reductive events: (a) Wu, F.; Lu, W.; Qian, Q.; Ren, Q.; Gong, H. Org. Lett. 2012, 14, 3044; (b) see also ref. 5 and 6.

(16) At present, we believe that electron-rich ligands such as $\mathbf{L 1 5}$ might prevent the binding of $\mathrm{CO}_{2}$ to the Ni center

(17) While the use of unactivated alkyl chlorides resulted in no conversion, the coupling of alkyl iodides delivered 14-19\% yield with significant amounts of dimerization events. All attempts to improve these results were not successful.

(18) The coupling of phenethyl electrophiles primarily resulted in dimerization with traces of styrene derivatives

(19) Dimerization and $\beta$-hydride elimination account for the mass balance. Although a screening was conducted for substrates with low yields, the results were not satisfactory.

(20) For selected examples: (a) Wu, J.; Hazari, N. Chem. Commun. 2011, 47, 1069. (b) Shi, M.; Nicholas, K. M. J. Am. Chem. Soc. 1997, 119, 5057, and citations therein

(21) For selected reviews dealing with $\mathrm{C}-\mathrm{O}$ electrophiles, see: (a) Mesganaw, T.; Garg, N. K. Org. Process Res. Dev. 2013, 17, 29. (b) Yamaguchi, J.; Muto, K.; Itami, K. Eur. J. Org. Chem. 2013, 19. (c) Rosen, B. M; Quasdorf, K. W.; Wilson, D. A.; Zhang, N.; Resmerita, A.-M.; Garg, N. K.; Percec, V. Chem. Rev. 2011, 111, 1346.

(22) The coupling of 1-bromo-4-(6-bromohexyl)benzene resulted in recovered starting material.

(23) The carboxylative event could be conducted in the dark with no erosion in yields, thus suggesting that light is not necessary for the reaction to occur.

(24) For recent metal-catalyzed hydrocarboxylation reactions: (a) Greenhalgh, M. D.; Thomas, S. P. J. Am. Chem. Soc. 2012, 134, 11900. (b) Williams, C. M.; Johnson, J. B.; Rovis, T. J. Am. Chem. Soc. 2008, 130, 14936.

(25) For related Pd-catalyzed events using isotopically-labelled intermediates: (a) Monks, B. M.; Cook, S. P. J. Am. Chem. Soc. 2012, 134, 15297. (b) Netherton, M. R.; Fu, G. C. Angew. Chem. Int. Ed. 2002, 41, 3910. (c) Stokes, B. J.; Opra, S. M.; Sigman, M. S. J. Am. Chem. Soc. 2012, 134, 11408.

(26) Care must bet taken when selecting the appropriate model substrate for isotope labelling. Whereas the $J_{l, 2}$ in $7 \mathbf{a}$ and $7 \mathbf{c}$ are significantly different (Scheme 3), other related $\gamma, \gamma$ unsubstituted alkyl bromide such as (5-bromopentyl)benzene had similar $J_{1,2}$ in both erythro and threo-isomers.

(27) In line with such hypothesis, we found that radical scavengers such as TEMPO or BHT inhibited the reaction. Furthermore, aliphatic alcohols are obtained as byproducts when employing alkyl tosylates, an observation that is consistent with a radical pathway. See for example: Madabhushi, S.; Kumar, B. A.; Narender, R. Tetrahedron Lett. 1998, 39, 2847; (b) Closson, W. D.; Wriede, P.; Bank, S. J. Am. Chem. Soc. 1966, 88, 1581, and citations therein.

(28) In the absence of $\mathrm{CO}_{2} 7$ a-d resulted predominantly in dimerization events and traces of $\beta$-hydride elimination.

(29) We cannot rule out that L14 and L16 act as a "redox-non innocent ligands". See for example: G. D.; Martin, J. L.: McFarland, C.; Allen, O. R.; Hall, R. E.; Haley, A. D.; Brandon, R. J.: Konovalova, T.; Desrochers, P. J.; Pulay, P.; Vicic, D. A. J. Am. Chem. Soc. 2006, 128, 13175.

(30) For the intermediacy of Ni species in odd oxidation states: (a) Refs. 5a, 5b, 6a. (b) Laskowski, C. A.; Bungum, D. J.; Baldwin, S. M.; Del Ciello, S. A.; Iluc, V. M.; Hillhouse, G. L. J. Am. Chem. Soc. 2013, 135, 18272. (c) Breitenfeld, J.; Ruiz, J.; Wodrich, M. D.; Hu, X. J. Am. Chem. Soc. 2013, 135, 12004. (d) Biswas, S.; Weix, D. J. J. Am. Chem. Soc. 2013, 135, 16192.

(31) For a mechanistic hypothesis, see Supporting information. 
Catalytic carboxylation of unactivated

alkyl bromides \& sulfonates

${\underset{\mathrm{H}}{\mathrm{H}=\mathrm{Br} \text {, OTs }} \mathrm{X}}_{\begin{array}{c}\text { Operationally-simple \& mild conditions } \\ \text { Exquisite chemoselectivity profile }\end{array}}^{\mathrm{CO}_{2}(1 \mathrm{~atm}), \mathrm{rt}} \begin{gathered}31 \text { examples } \\ \text { up to } 85 \% \text { yield }\end{gathered}$

up to $85 \%$ yield $\mathrm{R} \quad \mathrm{R}=\mathrm{Me}, \mathrm{L14} \mathrm{R}^{\mathrm{R}}$

Exquisite chemoselectivity pro

$\mathrm{R}=\mathrm{Me}, \mathbf{L 1 4}$
$\mathrm{R}=\mathrm{E} t, \mathbf{L 1 6}$

